\title{
TEC FOR HIGH POWER ELECTRONICS IN SPACE ENVIRONMENT
}

\section{KUMUD DARSHAN YADAV ${ }^{1}$, ULKESH B. DESAI ${ }^{2} \&$ ARUP K. HAIT $^{3}$}

${ }^{1}$ Department of Aeronautical and Automobile Engineering, Manipal Institute of Technology, MAHE, India

${ }^{2}$ Microwave Payload Mechanical Division, Space Applications Centre, ISRO, India

${ }^{3}$ Microwave and Antenna Mechanical Group, Space Applications Centre, ISRO, India

\begin{abstract}
The thermal management of satellites has become a major issue with the increase in the use of high power electronics. In this work, we demonstrate an unconventional method to reduce the hot spots present in high power electronic packages. The thermoelectric cooler (TEC) is designed for a specific structural and electrical constraints to cool down the high power amplifier to enhance its performance. Theoretical and Simulation work is presented to demonstrate the effectiveness of using TECs in such application. Further combined thermal - electric simulation of TEC embedded in the electronic package is shown to show the end effect of using TEC.
\end{abstract}

KEYWORDS: TEC, TEM, Thermal-Electric \& ANSYS

Received: Aug 11, 2018; Accepted: Aug 31, 2018; Published: Oct 16, 2018; Paper Id.: IJMPERDDEC201811

\section{INTRODUCTION}

The thermoelectric cooler otherwise known as solid-state pumps works on the principle of Peltier effect. The Peltier effect is an inverse of Seebeck effect which was discovered in the 1800s. When a certain amount of current is passed through the junctions of a conductor or semiconductor temperature difference can be observed as stated in [1]. The heat released at the hot junction is directly proportional to the amount of current supplied. These devices are highly reliable since they don't have any moving part and there is no fluid running inside them. These features make TEC a perfect candidate for the thermal management of electronic packages. Our work mainly focuses on demonstrating the use of TEC embedded inside the electronic packages for space application. A twostage thermoelectric is designed specifically for high power package and the schematic is shown in figure 1.

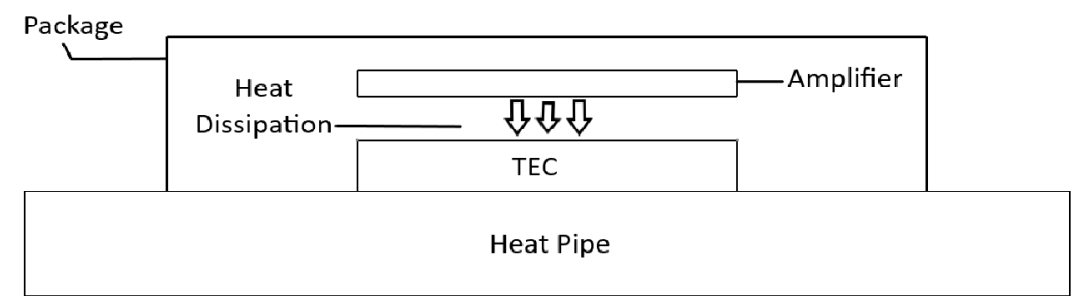

Figure 1: Schematic Representation of TEC Inside Package

\section{ANALYTICAL MODEL}

In this section, the analytical model of a two-stage thermoelectric cooler is presented. The current 'I' is supplied through the pellets for both stages which are connected electrically in series and thermally in parallel. Assumptions made for developing the equations: 
- The Thomson effect is considered to be negligible since it has almost no effect on the performance of TEC.

- The contact resistance between the thermoelectric leg, conductor and ceramic are negligible.

- The thermoelectric material properties are independent of temperature.

- The effect of convection and radiations are not taken into account.

A thermoelectric module with ' $n$ ' number of thermocouples with leg length ' $L$ ' and the cross-section area of each pellet 'A'. A steady-state 1-D model is presented of TEC. The Heat balance equation at the cold end takes the form after taking all assumptions into consideration given in [2][3].

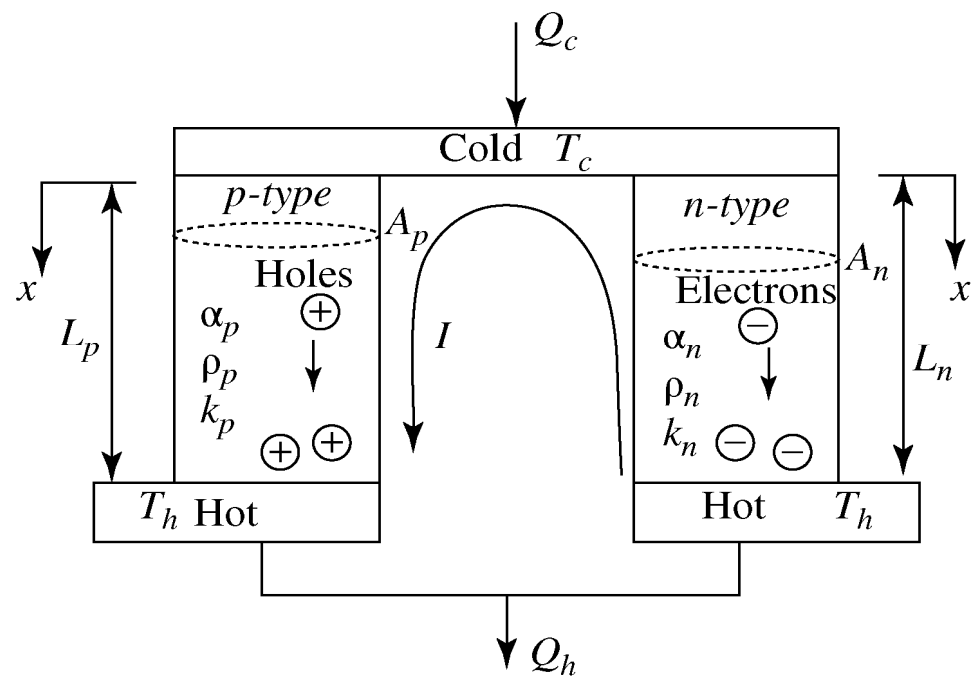

Figure 2: Working of the Thermoelectric Couple [2]

$\dot{Q_{c}}=q_{p}+q_{n}$

where $\mathrm{q}_{\mathrm{p}}$ and $\mathrm{q}_{\mathrm{n}}$ are heat flow in p-type and n-type semiconductor. The heat flow is given by Fourier's law of conduction and Peltier heat. Mathematically it is represented as

$\dot{Q}_{c}=n\left[\alpha T_{c} I-\frac{1}{2} I^{2} R-K\left(T_{h}-T_{c}\right)\right]$

Where,

$\alpha=\alpha_{p}-\alpha_{n}$

$\mathrm{R}=\frac{\rho_{p} L_{p}}{A_{p}}+\frac{\rho_{n} L_{n}}{A_{n}}$

$K=\frac{k_{p} A_{p}}{L_{p}}+\frac{k_{n} A_{n}}{L_{n}}$

Similarly, The heat flow at hot end $\mathrm{Q}_{\mathrm{h}}$ is given as

$\dot{Q_{h}}=n\left[\alpha T_{h} I+\frac{1}{2} I^{2} R-K\left(T_{h}-T_{c}\right)\right]$

Now, a two-stage thermoelectric cooler can be visualized as two single stage coolers stacked over one another as suggested in [4] with $\mathrm{n}$ number of thermocouples for 1 st stage and n0 for 2 nd. The thermoelectric cooler used in this 
research has different pellet cross-section area for each stage and area of the cold side surface is smaller than the area of the hot side. The heat flow from the hot side of the $1^{\text {st }}$ stage is considered as the heat flow into the cold side of the $2^{\text {nd }}$ stage. Since the geometrical configuration of pellets of the two stages is different the thermoelectric parameters for both stages will be different. The heat released from the 1 st stage is equal to the heat flow into the 2 nd stage with considering there is no another external heat load i. e.

$$
\dot{Q_{h_{1}}}=\dot{Q_{c_{2}}}
$$

Equating the above equation and heat balance equation at the cold end of the first stage we can solve for unknown parameters in our study. The two above equations result in

$$
\left(\alpha T_{m}+K\right) T_{c}-K T_{m}=\frac{Q_{c}}{n}+\frac{1}{2} I^{2} R
$$

And

$$
\begin{aligned}
& K T_{c}+\left[(\alpha I-K)-N\left(\alpha I+K^{\prime}\right)\right] T_{m}=-\frac{1}{2} I^{2} R-\frac{1}{2} I^{2} R^{\prime}-K^{\prime} T_{h} N \\
& N=\frac{n^{\prime}}{n}
\end{aligned}
$$

The results obtained are presented in the coming sections.

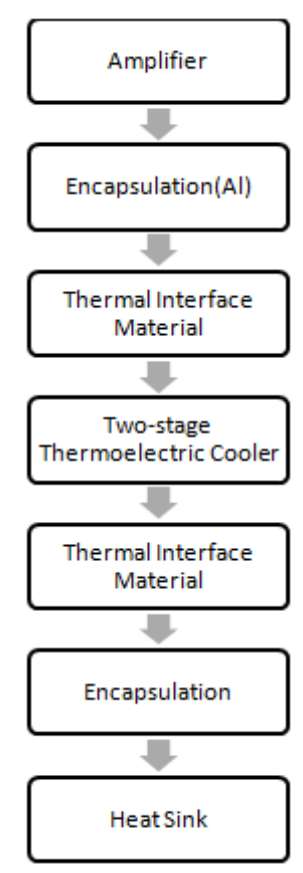

Figure 3: Heat Flow Diagram inside the Electronic Package

\section{MODELING OF TEC}

A two-stage thermoelectric module is modeled in Autodesk Inventor as can be seen in figure 4 . The thermoelectric pellets are joined using gold as a conductor in series and Aluminum Nitride as ceramic for the top, middle and bottom insulation. Aluminum nitride is used as ceramic since it has higher thermal conductivity and good insulation properties [5]. The semiconductor used for pellets based on ambient temperature is Bismuth $\mathrm{Telluride}\left(\mathrm{Bi}_{2} \mathrm{Te}_{3}\right)$. $\mathrm{The}$ properties used in calculation and simulation of TEC are of single crystal bismuth telluride given in [6]. 
The thermoelectric module consists of 30 thermocouples in $1^{\text {st }}$ stage and 36 thermocouples in $2^{\text {nd }}$ stage. The cold end of the module is smaller than the hot end due to the structural constraint. The pellets in the 1st stage have more cross section area than 2 nd stage as they have to withstand more heat load. This was concluded after a series of simulation with varying the cross-section area and the leg length of the pellet. The module is encapsulated inside 0.8 mm thick aluminum case to build redundancy in the system in case of outgassing.

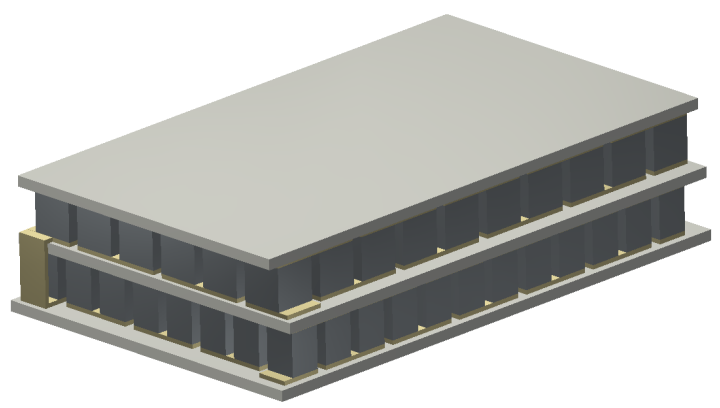

Figure 4: Two-Stage TEC CAD Design

Table 1: Module Specifications

\begin{tabular}{|l|c|c|}
\hline \multicolumn{1}{|c|}{ Geometrical Specifications } & Values & Units \\
\hline P-type and N-type pellets (1 $1^{\text {st }}$ stage) & & \\
\hline Cross Section Area & $2 \times 2$ & $\mathrm{~mm}^{2}$ \\
\hline Length & 2 & $\mathrm{~mm}$ \\
\hline P-type and N-type pellets $\left(2^{\text {nd }}\right.$ stage $)$ & & \\
\hline Cross Section Area & $2 \times 2$ & $\mathrm{~mm}^{2}$ \\
\hline Length & 2 & $\mathrm{~mm}^{2}$ \\
\hline Area of cold side & 378 & $\mathrm{~mm}^{2}$ \\
\hline Area of hot side & 433.44 & $\mathrm{~mm}^{2}$ \\
\hline
\end{tabular}

\section{SIMULATION}

The modules used in this research are the Steady-State thermal module and Thermal-Electric module. The first one is used for thermal analysis of the package itself while the latter one is used to simulate the working of TEC. Another simulation is also carried out in thermal-electric module here the TEC is incorporated in the package itself as shown in figure 1 . The boundary condition used for the simulation is given in table 2 .

The TEM was designed to remove the heat load of $25 \mathrm{~W}$ with sink temperature of $40^{\circ} \mathrm{C}$. A criterion was set from several iterations to bring the cold end temperature below $100^{\circ} \mathrm{C}$ to meet the desired requirements for high power electronic packages. The result for such setup is presented in figure 5. These high power packages dissipate a lot of heat which sometimes makes non-favorable conditions for certain components.

As seen from the hot-spots there are two high power amplifiers inside the package so TEC are placed right below the amplifiers by cutting out the material from the tray of the specified dimension. The clearance between the cold end and the upper face of the tray is $2 \mathrm{~mm}$ which is considerable for static stiffness. These main source of heat dissipation inside the package are high power amplifiers. However, boundary condition here is different from the previous TEC simulation.

Table2: Boundary Conditions for Simulation

\begin{tabular}{|c|c|}
\hline $\mathrm{T}_{\mathrm{h}}$ & $40^{\circ} \mathrm{C}$ \\
\hline $\mathrm{Q}_{\mathrm{c}}$ & $25 \mathrm{~W}$ \\
\hline $\mathrm{I}$ & $5 \mathrm{~A}$ \\
\hline
\end{tabular}




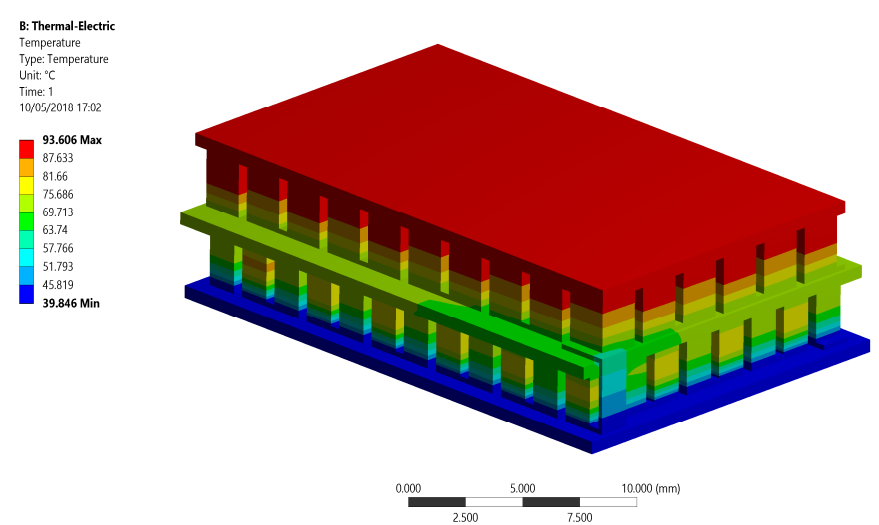

Figure 5: ANSYS Simulation of TEC at 5A

Table 3: Boundary Conditions for Combined Simulation

\begin{tabular}{|c|c|}
\hline $\mathrm{T}_{\text {sink }}$ & $60^{\circ} \mathrm{C}$ \\
\hline $\mathrm{Q}_{\text {amplifier }}$ & $95 \mathrm{~W}$ \\
\hline $\mathrm{I}$ & $5 \mathrm{~A}$ \\
\hline
\end{tabular}

Figure 6 depicts the temperature contour of the package when there is no TEC. The hot red regions depict the temperature due to heat dissipation from high power amplifiers. The temperature of the base rises to $110^{\circ} \mathrm{C}$ which results in the rise of the junction temperature of the amplifier.

The junction temperature of the amplifier is the driving key to deploy an active cooling system inside the electronic package. After incorporating the TEC inside the electronic package the results were very promising as can be seen in Figure 7. A significant decrease in temperature is observed at the base where temperature reduces to $85.5^{\circ} \mathrm{C}$. The designed TEC completely suits the requirement of the electronic package for optimum performance and also increases the reliability of the amplifier by maintaining its junction temperature.

\section{RESULTS AND DISCUSSIONS}

Figure 8 depicts the comparison between the analytical results and results obtained from ANSYS simulation. This shows a fair resemblance in the results obtained which validates that the simulation results are in accordance with the analytical model. The results obtained show very less deviation from ideal results even the simulation does consider the contact resistance factor into consideration. This happens because the leg length of the thermocouples used is big to nullify the variation caused by contact resistance. This can further be validated from [7].

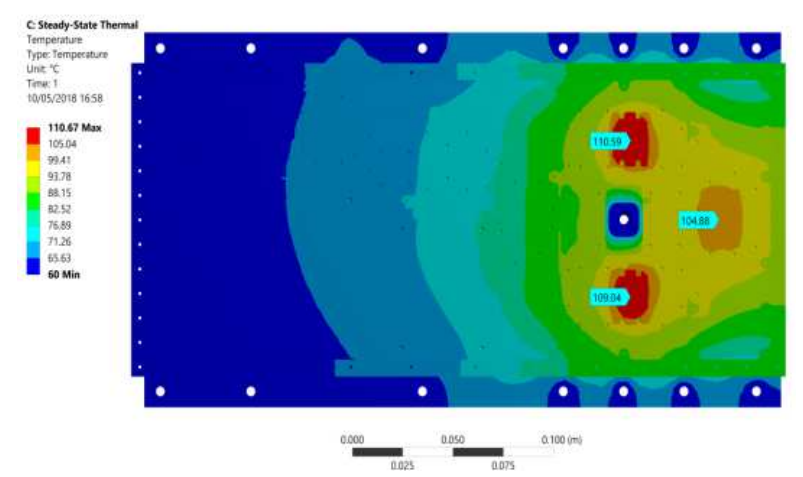

Figure 6: Temperature Contour of the Tray before TEC is Placed 


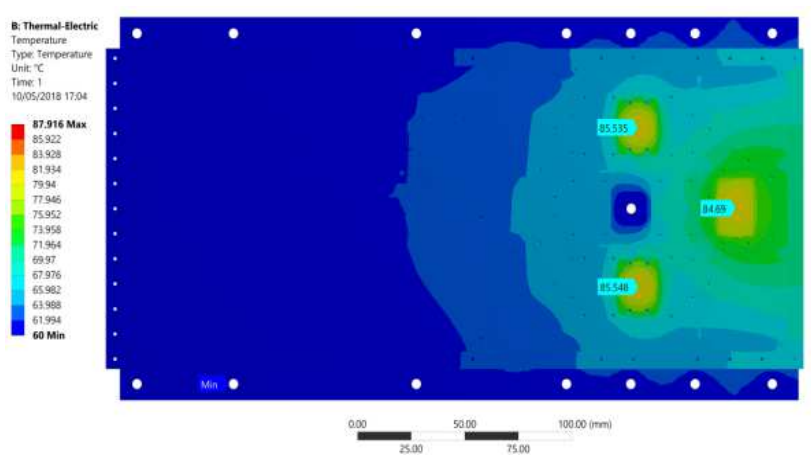

Figure 7: Temperature Contour of the Tray after TEC is Placed

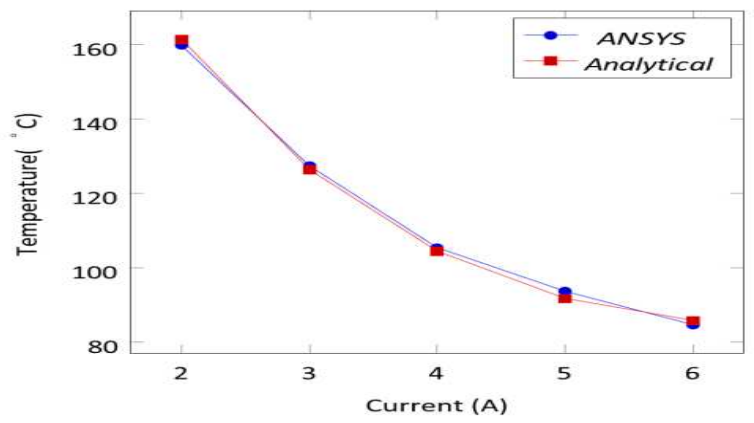

Figure 8: Comparison of Results from Simulation and Ideal Equation

\section{CONCLUSIONS}

An anomaly is observed during the simulation process when the heat starts building up inside the pellets when current is supplied more than 5 amps. This phenomenon can be explained by the Joule's heat. Since all conductors and semi-conductor exhibit the joule's effect, the region from 5 amps to 6 amps is where the joule's effect becomes dominant over the thermoelectric effect. So, the maximum current which can be supplied to the module to observe thermoelectric effect is near 5 amps.

\section{REFERENCES}

1. A. Attar, "Studying the optimum design of automotive thermoelectric air conditioning," Kalamazoo, 2015.

2. H. Lee, Thermoelectrics Design and Materials. John Wiley and Sons, 2016.

3. N. Korprasertsak and T. Leephakpreeda, T, "Mathematical modeling and validation of cooling/heating effects in thermoelectric module coupled with heat sinks," IEEE, 7th International Conference on Mechanical and Aerospace Engineering, 2016.

4. R. Yang, G. Chen, G. J. Snyder, J. P Fleurial, “Multistage thermoelectric microcooler,” Journal of Applied Sciences, 2004.

5. Kumar, B., Reddy, K. H., \& Kumar, C. R. V. Significance Of Finite Element Method Based Modeling In Analysing Temperature Generation And Distribution During Hard Turning.

6. P. Fleurial, A. Borshchevsky, M. Ryan, W. Phillips, E. Kolawa, T. Kacisch, and R. Ewell, "Thermoelectric microcoolers for thermal management applications,” 16th International Conference on Thermoelectrics, IEEE, 1997.

7. H. Lee, Thermoelectrics Handbook - Macro to Nano. CRC Press Taylor and Francis Group, 2006.

8. P. I Mani, “Design, modeling and simulation of a thermoelectric cooling system (tec), master's theses. 749,” 2016. 